\title{
Multimedia Streaming in Wireless Ad Hoc Networks According to Fuzzy Cross-layer Approach
}

\author{
Maryam Ravaei \\ Department of Computer \\ Science and Research Branch \\ Islamic Azad University, Kish, Iran
}

\author{
Javad Mirabedini \\ Department of Computer \\ Islamic Azad University \\ Central Tehran Branch, Iran
}

\author{
Ali Harounabadi \\ Department of Computer \\ Islamic Azad University \\ Central Tehran Branch, Iran
}

\begin{abstract}
Nowadays, with progress of technology in wireless communications and increase of wireless tools to establish communication, and for sending and receiving data files especially multimedia files, ad hoc networks have received special attention. Mobile communications are usable wherever communication infrastructure is limited or absent, or establishing an infrastructure is not affordable. According to the ever increasing usage of these networks, in this article, attempts have been made to select some routs for sending multimedia files in an ad hoc network by using fuzzy logic and paying attention to moving nodes, so that we can fulfill needs for service quality in this kind of networks.
\end{abstract}

Keywords: Wireless Ad Hoc Networks; Fuzzy logic; Multimedia Streaming

\section{INTRODUCTION}

A mobile ad hoc network (MANET) is a special kind of infrastructure less network. The word "ad hoc" stems from Latin and means "for this purpose" which connotes "temporary". The reason for such naming is that these networks are established temporarily, especially for places such as battle fields that have no stable infrastructure or need a fast establishment of networks.

MANETs include wireless devices that together create a selforganizing network. These networks have no stable communication infrastructure. Since the range of sending wireless mediums is limited, communications in these networks depends on middle nodes. Therefore, each node also plays the role of a router in networks. In these networks, network topology is always changing, and the reason is mobility of network nodes. Additionally, new nodes may be added to or omitted from the network at any time, or some nodes may turn themselves off. There is no stable central control for backing up network configuration or reconfiguration.

MANETs have extensive applications in military networks, since they are dynamic, have an inherent mobility, and do not need stable communication infrastructures. The first usages of MANETs go back to DARPA,PRNet project in 1972 for transferring radio packets.

PRNet established a radio network with the least central control in which a combination of CSMA and Aloha was used as a protocol for accessing media. This network provided the possibility for establishing connections among a couple of users in a big geographical area.

In 1983,SURAN appeared as a developed form of DARPA in terms of scalability, security, and energy management. In late 80 s and early $90 \mathrm{~s}$, the fast growth of the internet and minicomputers fulfilled the idea of using data transmission by packages in radio networks.

In early 90s, fast development of technology created a new phase for MANETs. The IEEE 802.11 committee accepted the term Ad Hoc Network which had a non-military and noncommercial meaning. In late 90s the group MANET started working in IETF and standardized the protocols of routing in MANETs. Also, the IEEE 802.11 committee started the standardization of protocols for accessing media based on minimizing collision and increasing fault tolerability.

These networks are highly flexible and suitable for different programs and situations. Therefore, establishing temporary communications in them does not need a predetermined structure. Because of the limited transmission range of wireless mediums, traffic is relayed between a couple of middle notes for establishing connections between two far away nodes; therefore, these networks are also sometimes called mobile multihop ad hoc networks.

In the field of MANETs a lot of research has been conducted some of which will be mentioned. 
You et al. proposed an exploration routing protocol based on hop counts. In their study, an exploration function has been used to assist routing. $\mathrm{Vu}$ et al. [2] suggested an on-demand protocol considering the mobility in nodes and capability of detecting local faults.

Sivakumar et al. [3] proposed a protocol based on machine learning with an optimal energy consumption approach via load balancing in critical nodes. Xia et al. [4] introduced a flexible trust-based on-demand routing protocol for selecting the shortest and safest route for transmission of data packages in MANETs.

Mousaoui et al. [5] suggested a new mechanism for creating stable routes between pairs of network nodes on the OLSR routing protocol. Kumar et al. [6] proposed a model for managing energy and controlling energy consumption by decreasing the time of data transmission in MANETs.

Sridhar et al. [7] introduced an energy-based protocol on the known protocol AODV which, based on send/receive rates of nodes and data size, calculates the energy level of nodes before selecting a node for routing, and decides if it is enough for transmission . Sumathia et al. [8] implemented Adaptive HELLO messaging scheme on the dynamic on-demand protocol which uses connectivity information and local links for observing conditions of links between nodes in order to decrease energy consumption.

Mousaoui et al. [9] suggested a mechanism for creating highly stable, fault-tollerant paths among all mobile nodes in MANETs; in their suggested method, a stability function was used in OLSR protocol to calculate the mobility degree of a node compared with its neighbors to select routes with the least amount of mobility. Kanakaris et. al [10] investigated efficiency of protocols in MANETs named AODV, DSDV, and DSR, by considering the factor of mobility in nodes on different network scales.

Xia et al. [11] proposed an on-demand trust-based uni-cast routing protocol for choosing the shortest routs according to needs for security in data packets transmission for routing of MANETs. Jain et al. [12] introduced different proposals for energy level accuracy in order to decrease energy loss and to increase data transmission and networks' life time.

Tabatabei et al. [13] proposed a new reward-based protocol in order to increase throughput and provide service quality for
MANETs. Palma et al. [14] suggested a new protocol for MANETs that selects routes according to the situation of links in order to increase network efficiency.

In this article, we have presented a routing protocol for multimedia streaming in MANETs according to a fuzzy cross-layer approach. In the second part, the operation of this protocol will be explained; in the third, simulation results will be presented; and in the fourth, conclusion will be derived.

\section{BACKGROUND}

\subsection{Scheduling Method}

In the suggested protocol, fuzzy logic will be used for routing multi-media files, which will be briefly explained.

Each type III fuzzy system includes four main parts: fuzzy rule base, fuzzy inference engine, fuzzifier, and defuzzifier (Figure 2.2). Each part will be reviewed below.

Fuzzy rule base is a collection of fuzzy if-then rules. It is considered the heart of fuzzy systems, since other components of fuzzy systems are used for establishing these rules in an effective and efficient totality. As mentioned before, the fuzzy rule base includes if-then rules shown below:

If $X_{1}$ is $A_{1}, X_{2}$ is $A_{2}, \ldots$ and $X_{n}$ is $A_{n}$, then $y$ is $B$.

In this rule, As and B are fuzzy sets; and Xs and y are linguist variables of input and output in a fuzzy system.

A fuzzifier can be considered as a mapping from a real-valued point to another point in a fuzzy set of inference engine.

In the suggested protocol of fuzzy system input, four parameters have been considered: hop count, movement speed of nodes, available bandwidth, and energy at battery level of nodes.

For each input variable, two fuzzy sets having trapezoidal membership functions will be defined ( $\mathrm{H}$ stands for high level, and 1 for low level), shown in Figures a1-2, b2-2, d3-2, and e42. Trapezoidal membership functions are used because they are accurate. For output or stability rate of link, four fuzzy sets with triangular membership functions ( $\mathrm{H}$ for high level, $\mathrm{VH}$ for very high level, $\mathrm{M}$ for moderate, $\mathrm{L}$ for low level, and VL for very low level) have been used which are shown in Figure 6-5. 


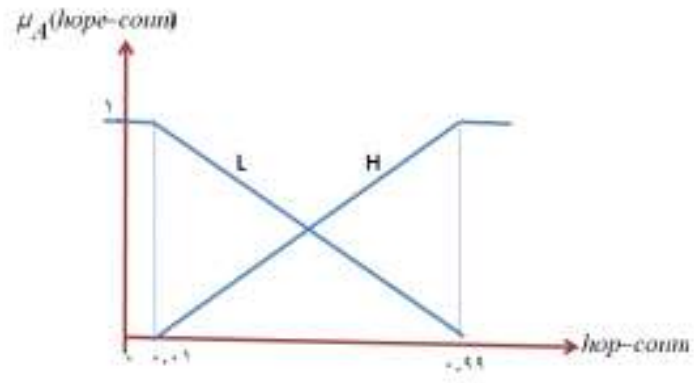

Figure a-2-1. Membership functions for input variables of hop count

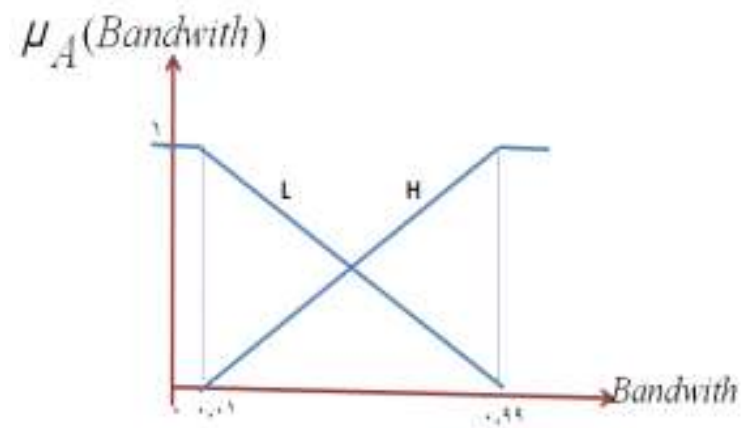

Fig ure b-2-2. Membership functions for input variables of bandwidth

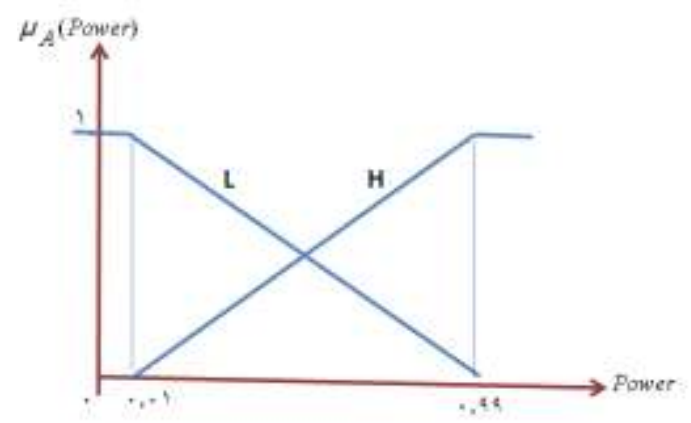

Figure C-2-3. Membership functions for input variables of energy on battery level

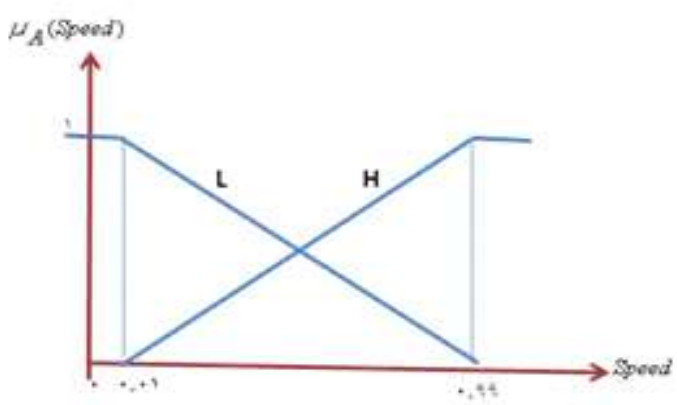

Figure d-2-4. Membership functions for input variables of movement speed in nodes

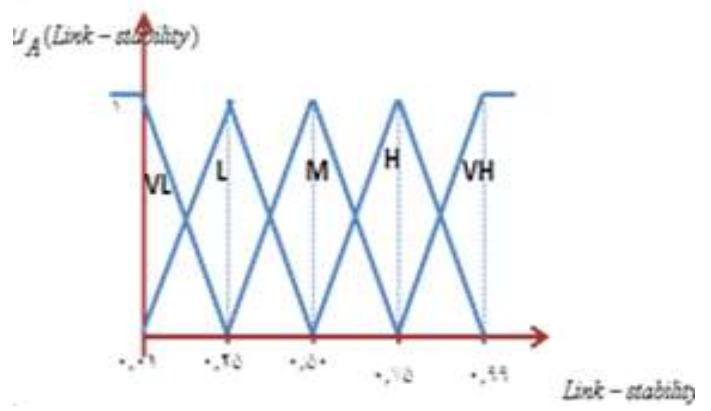

Figure e-2-5. Membership functions for output variables of stability rate or link desirability

\section{PREVIOUS RESEARCH}

Using fuzzy rules in inference stage, link stability is calculated according to the value of considered parameters, that is, hop counts, movement speed, available bandwidth, and energy at battery level of nodes. Each fuzzy rule includes two parts; one is introduction: "if energy at battery level is low, hop count is high, bandwidth is low, and the amount of movement in nodes is low", and the other part is conclusion: "then the link stability is low". In the suggested method, fuzzy inference engine has been considered as minimum Mamdani.

For each of the four input parameters, two fuzzy sets have been defined, so 16 fuzzy rules are obtained. These 16 fuzzy rules are defined in Table1.

Table 1. Sixteen Fuzzy Rules

\begin{tabular}{|c|c|c|c|c|c|}
\hline No. & \multicolumn{4}{|c|}{ Inpest } & Ontput \\
\hline$i$ & $\begin{array}{l}\text { Hepe } \\
\text { count }\end{array}$ & $\begin{array}{l}\text { Arailable } \\
\text { baudwidth }\end{array}$ & $\begin{array}{c}\text { power } \\
\text { remainiag }\end{array}$ & $\begin{array}{l}\text { Mobility } \\
\text { speed }\end{array}$ & $\begin{array}{c}\text { Liak } \\
\text { rabaisy }\end{array}$ \\
\hline 1 & Low & Lew & tow & Lent & Mrdivat \\
\hline 7 & Lau & tor & tew & Biab & 200 \\
\hline+ & Law & Len & Hiat & $2 \partial \%$ & Hipht \\
\hline 4 & Kaw & Lew & Bigh & Bight & Hight \\
\hline 2 & Law & Hight & Las & Law & Hight \\
\hline 5 & Lar & Bigh & Les & Elab & Medive \\
\hline $\mathrm{F}$ & Inom & High & Hiak & Law & Two High \\
\hline 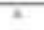 & Iaw & High & High & High & Hight \\
\hline 1 & Figh & Iew & Lat & Iaw & Ies \\
\hline W. & Bigit & Lees: & Laut & Bigi & Tary Lan \\
\hline 11 & Bngh & $20 \%$ & स्ight & Lan & Mediant \\
\hline 19 & Hipit & Low & Higi & Figh & Eor \\
\hline 17 & Figb & High & Low & Law & Mediant \\
\hline 11 & $B g 6$ & Bhot & toos & High & tea \\
\hline 10 & घhat & Bipl & $m, z^{i}$ & 200 & "High \\
\hline 19 & Bigh & Hiph & Biph & Hiah & Melian: \\
\hline
\end{tabular}




\subsection{Defuzzification Stage}

To translate fuzzy output into numeric values, defuzzifier is used. In the suggested method, center average defuzzifier has been used.

In the suggested protocol, the following stages will be used for routing.

1. A source node checks its routing table for finding a rout to destination. If it has an available route with needed energy range or the minimum energy needed for sending data or file, it uses that route to send data packages.

2. If there is no route, the source node starts a process of route detection and creates a package of route request with the address of destination node and its parameters; then casts the package of route request in the public network.

3. Through the route, each middle node which receives the package of route request, first extracts the data inside it, and then, using fuzzy algorithm, determines suitability of the link received via the package. In other words, it checks if the amount of energy on battery level is enough in that node for sending. If there is enough energy, a positive score is considered for the node; otherwise, a negative score will be considered. The same will be done for other mentioned parameters as well. For example, it checks, and if there is enough bandwidth for sending the package, it receives a positive score; otherwise, it gets a negative score. Finally, if a suitable link is identified, or the sum of calculated scores is high, the adjacent connection table in the middle node will be updated.

4. Furthermore, the middle node checks if there is a route to destination in its routing table. If there is one, a package of route response is created; it enters its parameters together with the sum of scores into the package and sends it backward. If there is no route, it will publically recast the package of route request by entering its parameters and sum of scores.

5. When the package of route request reaches the destination node, it extracts the parameters and sum of scores from the route request package, just like the middle nodes did, and uses RL algorithm to determine suitability of links; if the link is suitable (the highest scores it has obtained form that route), it will be saved.
6. The destination node creates a package of route response, and extracts the fields of source and destination addresses directly from the package of route request, and copies them to the package of route response; it also adds its own parameters and sum of scores to it, and then sends the package of route response toward the source.

7. Each node, through the reverse route, extracts parameters and enters those of its own in the response package to determine suitability of links.

8. Source node sends data to destination using the discovered route.

\section{SIMULATION RESULTS}

In this article, for simulating the suggested protocol of fuzzy system and comparing it with AODV protocol, OPNET Modeler 10.5 simulation software has been used. The structure and instructions for installation of the software are mentioned below.

For simulation, after running OPNET 10.5, from the 'Scenario' menu, 'Scenario Components', 'Import', we have entered 'OPNET Scenarios', and selected 'MANET-AODV Streammobility-Scenario-with-Demands'.

Using 'Duplicate scenario' from 'Scenario' menu, 'AODV Stream' has been copied and the new scenario has been named 'Fuzzy System'.

In both scenarios of 'AODV Stream' and 'Fuzzy System', one variable has been defined for each node which determines for a node to use a standard 'AODV Stream' or 'Fuzzy System' protocol. This variable has been disabled for 'AODV Stream' scenario nodes, and enabled for 'Fuzzy System' scenario nodes. As seen in Figure 4.6, the simulated topology includes 25 nodes which have been distributed on an area of $1127 \mathrm{~m}$ by $1127 \mathrm{~m}$. For the model of nodes' movement, the tragedy was first changed to "Vector" model to move in a vector form, and a random cyclical mode from 0 to 359 degrees has been defined for each node. The time of cycle and movement speed of each node have been considered $0-200 \mathrm{~s}$ and $1-10 \mathrm{~m} / \mathrm{s}$ respectively. Figure 4.7 shows a view of node's editor. 


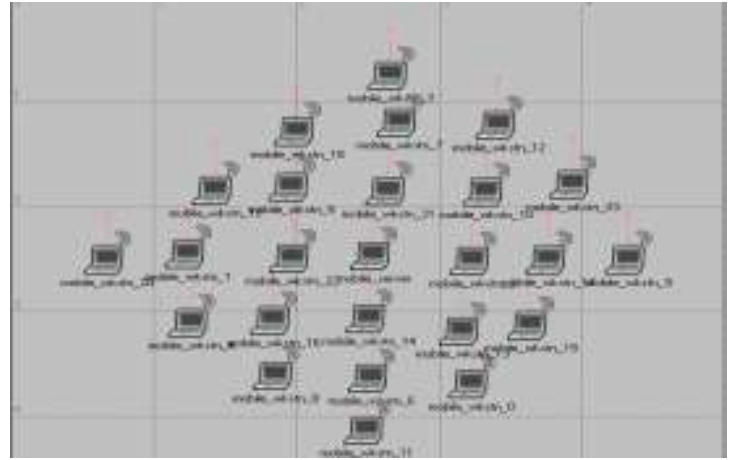

Figure 4.1. A view of network topology for the simulated mode

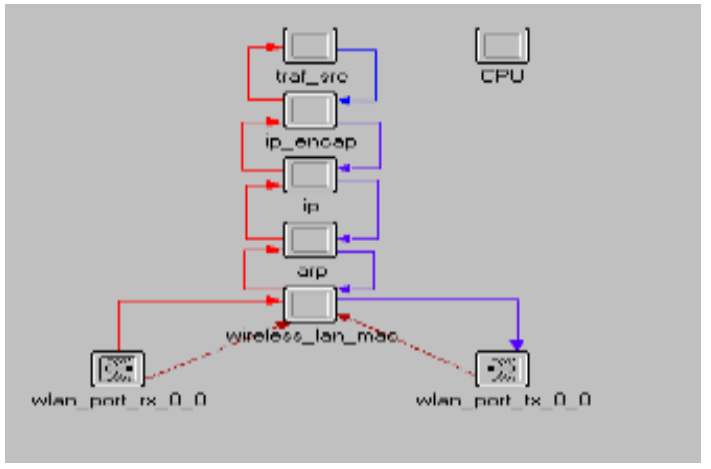

Figure 4.2. A view of node's editor for the simulated model

The transmission rage of each node is $250 \mathrm{~m}$; bandwidth has been randomly considered 1-10 Mbps; and the simulation time is $600 \mathrm{~s}$.

To make changes in the process model, 'Open' is selected from 'File' menu and then 'Process model' is selected for the type of files. By opening 'AODV Stream -rte' file form 'manet' folder, a diagram of mode with two modes of 'init' and 'wait' are observed which had already been available. A mode named 'TURN' has been added which is responsible for circulation of nodes in an expressed form. This process model is shown in Figure 4.3.

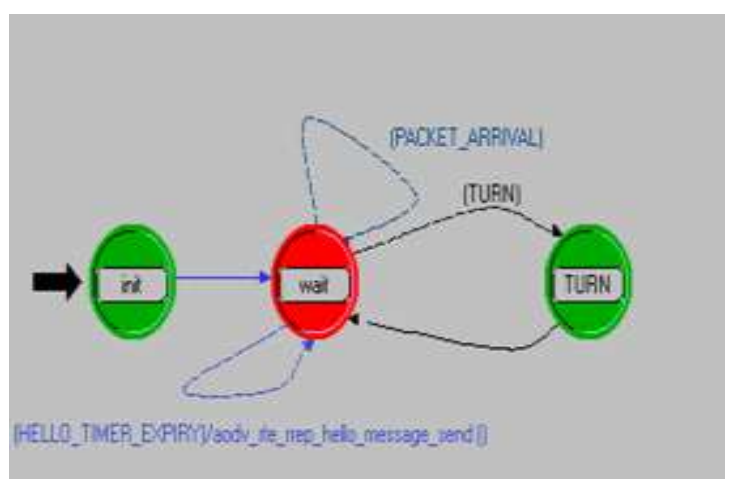

Figure 4.3. A view of processing model of simulated model
To see the code, the 'Edit Function Block' button is used. The main changes in codes for obtaining the suggested protocol includes calculating amounts of available bandwidth, energy level, node speed, hop count, radio frequency, antenna pattern field, signal productivity, and the level of radio sensitivity, and sending them to evaluation functions of reinforcement learning before updating the table in the functions: 'aodv_rte_rreq_pkt_arrival_handle' and 'aodv_rte_rrep_pkt_arrival_handle' which are respectively the function of carrying package of route request and the function of carrying the package of route response.

The Fuzzy System software package includes two folders named 'manet' and 'op-model'; To run the simulated model, the contents of 'manet' folder must be copied in 'manet' folder in:

\section{'C:IProgram Files\OPNET\A\models $\backslash$ std $\backslash$ manet10.5'}

and then the contents of 'op_model' must be copied in:

\section{'C:LDocument and Settingsluserlop_models'}

After running 'Opnet', we should select 'Edit' menu, enter 'preferences', and copy the address below to the existing paths:

'C:IDocument and

Settingsluserlop_models\Reinforciment_AODV Stream'

Now, we can run 'Fuzzy System' project from 'Fuzzy System' folder by going to 'File' menu and choosing 'Open'.

After simulation, diagram of results are observable in different forms using the 'View Result' button.

The number of package losses in the network is because they have no access to a route toward destination. Over time, 'Fuzzy' has fewer losses of package compared with 'AODV'. In Figure 4.9 the numbers of package losses are demonstrated.

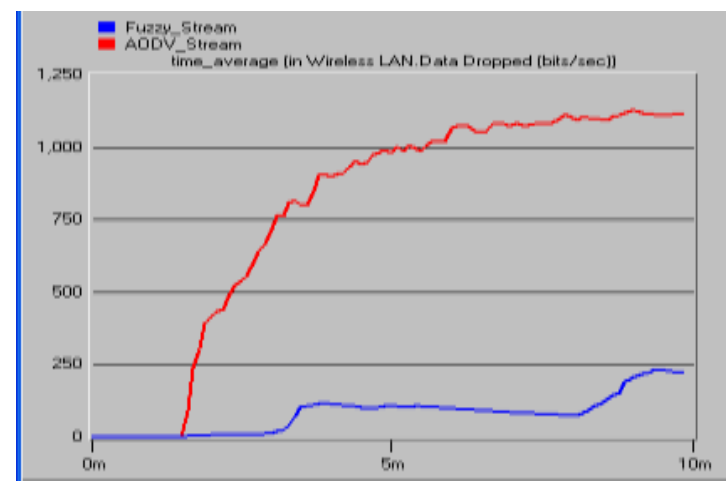

Figure 4.4. A view of the numbers of package losses 
Mean time is calculated from the start of transmission of package from the source node until the time the package is delivered to a destination. A remarkable reduction in delay parameter in wireless ad hoc network is received for 'Fuzzy' compared with 'AODV'. By creating more stable routes, in other words, a couple of routes are created which are likely to take packages to destination, and there is no need for rediscovering the route. In Figure 4.10, the total delay in the network is shown.

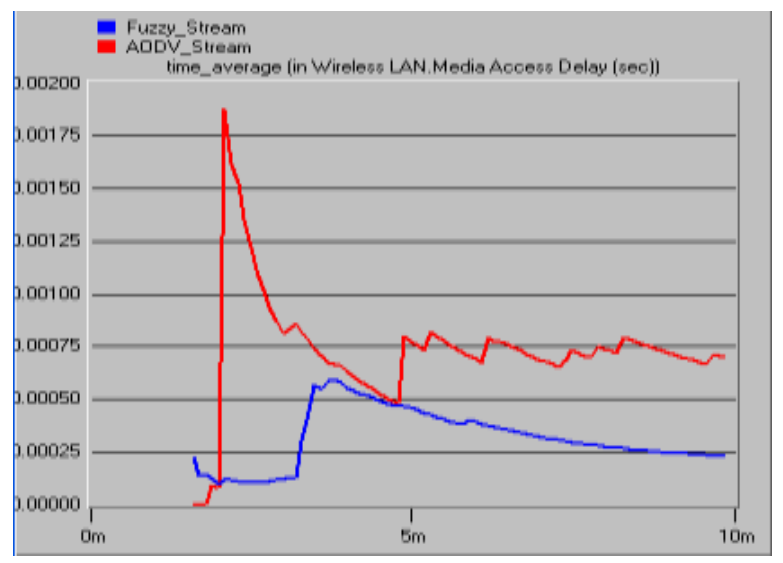

Figure 4.5. A view of total delay in the network

Compared with the total packages transferred by source nodes, the number of packages successfully delivered to destination is more in 'Fuzzy' than in 'AODV'. In Figure 4.11 transmission or delivery rate of packages are shown.

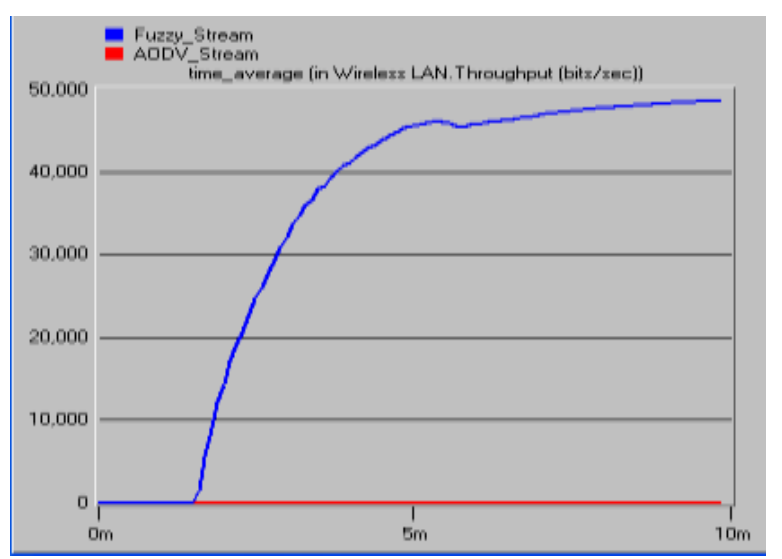

Figure 4.6. Transmission or delivery rate

\section{DISCUSSION}

In the suggested protocol of fuzzy system input, four parameters have been considered: hop count, movement speed of nodes, available bandwidth, and energy at battery level of nodes.

. Each fuzzy rule includes two parts; one is introduction: "if energy at battery level is low, hop count is high, bandwidth is low, and the amount of movement in nodes is low", and the other part is conclusion: "then the link stability is low". In the suggested method, fuzzy inference engine has been considered as minimum Mamdani. Mean time is calculated from the start of transmission of package from the source node until the time the package is delivered to a destination. A remarkable reduction in delay parameter in wireless ad hoc network is received for 'Fuzzy' compared with 'AODV'. This suggested protocol considers two important criteria for accessing more stable routes, and tries to create optimal routes to destination and increase their availability by creating routes with high available bandwidth and higher remained energy levels. In other words, it creates more stable routes for later more effective usages.

\section{CONCLUSIONS}

In this article, wireless ad hoc networks and their features were first introduced, and then their problems were reviewed. Types of routing protocols in wireless ad hoc peer-to-peer networks have been discussed. A new routing protocol named 'Fuzzy' has been presented for wireless ad hoc peer networks, whose efficiency was investigated in two faulty and non-faulty states. It increases efficiency of AODV protocol using cuckoo learning method with a high calculative speed.

This suggested protocol considers two important criteria for accessing more stable routes, and tries to create optimal routes to destination and increase their availability by creating routes with high available bandwidth and higher remained energy levels. In other words, it creates more stable routes for later more effective usages.

\section{REFERENCES}

[1] L. You, J. Li, C. Wei, C. Dai, J. Xu, L. Hu, "A hop count based heuristic routing protocol for mobile delay tolerant networks", Hindawi Publishing Corporation, Scientific World Journal, 2014.

[2] Vu TK, Kwon S, "Mobility-Assisted on-Demand Routing Algorithm for MANETs in the Presence of Location Errors", 
Hindawi Publishing Corporation, Scientific World Journal, 2014.

[3] B. Sivakumar, N. Bhalaji, D. Sivakumar, "A survey on investigating the need for intelligent power-aware load balanced routing protocols for handling critical links in MANETs", Hindawi Publishing Corporation, Scientific World Journal, 2014.

[4] H. Xia, Z. Jia, X. Li, L. Ju, EHM. Sha, “Trust prediction and trustbased source routing in mobile ad hoc networks", Ad Hoc Networks, 2013.

[5] A. Moussaoui, F. Semchedine, A. Boukerram, "A link-state QoS routing protocol based on link stability for Mobile Ad hoc Networks", Journal of Network and Computer Applications, 2014.

[6] K. Kumar, VP. Singh, "Power consumption based simulation model for mobile ad-hoc network", Wireless Personal Communications, 2014.

[7] S. Sridhar, R. Baskaran, P.Chandrasekar "Energy supported AODV (EN-AODV) for QOS routing in MANET", Social and Behavioal Science, 2013.

[8] A.Moussaoui, F.Semchedine, A.Boukerram "A link-state QoS routing protocol based on link stability for Mobile Ad hoc Networks", Journal of Network and Computer Applications, 2015 .

[9] V. Kanakaris, D. Ndzi, D. Azzi, "Ad-hoc Networks Energy Consumption: A review of the Ad-Hoc Routing Protocols", Journal of Engineering Science and Technology Review, 2010.

[10] H. Xia, Z. Jia, X. Li, L. Ju, EHM. Sha, "Trust prediction and trust-based source routing in mobile ad hoc networks", Ad Hoc Networks, 2013.

[11] Y. Kumar Jain, R. Kumar Verma, "Energy Level Accuracy and Life Time Increased in Mobile Ad-Hoc Networks Using OLSR", International Journal of Advanced Research in Computer Science and Software Engineering, 2012.

[12] Sh. Tabatabaei, M. Teshnehlab, S.J.Mirabedini, “A New Routing Protocol to Increase Throughput in Mobile Ad Hoc Networks", Wireless Personal Communications, 2015.
[13] D.Palma, M.Curado, "Onto scalable Ad-hoc networks: Deferred Routing”, Computer Communications, 2012. 